\title{
Asociación del índice predictivo de asma y presencia de la enfermedad en niños de la comuna de Valdivia
}

\author{
EVELYN KRAUSE G.*, KARIN GROB B.**, \\ MAURICIO BARRÍA P.*** y MARIO CALVO G.****
}

\begin{abstract}
Association of the asthma predictive index and presence of the disease in children in Valdivia, Chile
\end{abstract}

There are predictive indexes to identify asthmatic patients from the rest of the recurrent wheezing phenotypes. The aim of this work was to evaluate the association between the positive Asthma Predictive Index (API) and the presence of asthma between the age of six and seven years old, in children from Valdivia, Chile. Methods: API was applied to 101 asthmatic children (cases) and 100 non-asthmatic children (controls). Data were analyzed using STATA v.11 (2009). Fisher Exact Test was used to determine the relationship between variables. Results: $72.3 \%$ of asthmatic patients and $3 \%$ of non-asthmatic patients had a positive index. Significant differences $(p<0.001)$ were shown in all the variables included in the API. In our group of patients, the probability for a child to develop asthma was at least 24 times higher if he/she had a positive API $\left(O R=84.3 C_{95 \%} 24.1-436.5\right)$. Conclusion: $A P I$ is a good tool to predict asthma and allows to take right decisions in recurrent wheezing children younger than 36 months old.

Key words: Asthma, child, risk, score, wheezing.

\section{Resumen}

Es un desafio identificar pacientes asmáticos entre sibilantes recurrentes, por eso existen índices predictivos. El objetivo de este estudio es evaluar la asociación entre índice predictivo de asma (IPA) positivo, y presencia de asma entre los seis y siete años de edad, en niños de la comuna de Valdivia. Material y Método: Se aplicó el índice IPA a 101 casos (niños asmáticos) y 100 controles (niños sin asma). Se utilizó STATA v.11 (2009), y Test exacto de Fisher para determinar relación entre variables. Resultados: $72,3 \%$ de los pacientes asmáticos y 3\% de los no asmáticos tuvieron un índice IPA positivo. Se demostraron diferencias significativas $(p<0,001)$ en todas las variables que componen los criterios del IPA. En nuestros pacientes, la probabilidad de desarrollar asma fue al menos 24 veces

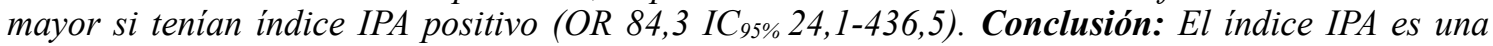
buena herramienta para predecir asma, y permite tomar decisiones acertadas en pacientes sibilantes menores de tres años.

Palabras clave: Asma, riesgo, niño, sibilancias.

\section{Introducción}

El asma infantil es una enfermedad inflamatoria heterogénea con diferentes fenotipos que dependen de la edad, género, antecedentes genéticos y exposición ambiental, y que siguen una vía común caracterizada por cuadros recurrentes de obstrucción de vía aérea ${ }^{1}$.

La mayor parte de los casos de asma comienza en los primeros años de vida, por lo que identificar niños con alto riesgo de desarrollar la enfermedad es una prioridad de salud pública ${ }^{2}$.

* Pediatra, Instituto de Pediatría, Universidad Austral de Chile (UACh).

** Pediatra Broncopulmonar, Sub-departamento de Pediatría, Hospital Base de Valdivia.

*** Enfermero, PhD en Salud Pública. Instituto de Enfermería, UACh.

**** Pediatra Broncopulmonar, Instituto de Pediatría, Universidad Austral de Chile. 
En Chile las consultas por obstrucción bronquial en atención primaria constituyen el $23 \%$ de las atenciones de morbilidad en menores de 15 años y según datos del Ministerio de Salud generan el $16 \%$ de todas las consultas en el grupo entre $5 \mathrm{y}$ 14 años ${ }^{3}$.

La presencia de sibilancias recurrentes en los primeros años de vida ocurre aproximadamente en el $40 \%$ de los niños, aunque sólo el $30 \%$ de los niños con sibilancias recurrentes tendrá asma a los seis años de edad ${ }^{4}$.

Estudios realizados en distintos países desarrollados, revelan un aumento sostenido de la prevalencia de asma y atopia en las últimas cuatro décadas ${ }^{5}$. En la búsqueda por predecir si un paciente sibilante padecerá asma, se han desarrollado índices predictivos. El Índice API (Asthma Predictive Index) o IPA, fue publicado por Castro-Rodríguez en el año $2000^{2}$, en base a la población evaluada en el Tucson Children Respiratory Study. Se trata de un índice predictivo de asma en aquellos niños menores de tres años con sibilancias recurrentes. Se compone de seis preguntas divididas en criterios mayores y menores. Los niños con IPA positivo tienen siete veces más posibilidad de tener asma en edad escolar que los con IPA negativo ${ }^{1}$ (Figura 1).

En base a los antecedentes es nuestro interés evaluar la utilidad del índice IPA en la comuna de Valdivia.

\section{Marco teórico}

El International Study on Asthma and Allergies in Childhood (ISAAC) es un proyecto que lleva más de 20 años de investigación, desarrollado con 1,96 millones de niños. Su objetivo es investigar asma, rinitis y eczema en la infancia. Este estudio demuestra que las cifras de prevalencia de asma varían mucho en distintos países ${ }^{6}$. En Chile, 10 a $15 \%$ de los niños en edad escolar son asmáticos, se considera un promedio de $12 \%{ }^{5}$.

En Chile, existen tres centros oficiales del estudio ISAAC (Santiago, Valdivia y Punta Arenas), evaluados en fase I (1992) y fase III (2002). Se determinó la prevalencia de síntomas de asma en escolares chilenos entre dichas fases, a las edades entre seis y siete años, y entre trece $y$ catorce años ${ }^{4,7}$. En Valdivia en el año 2002, en el grupo etario entre seis y siete años, $49,3 \%$ de los pacientes tuvo sibilancias alguna vez, $15,7 \%$ diagnóstico de asma alguna vez, y un 21,4\% tuvo sibilancias en los últimos 12 meses, considerado como asma actual ${ }^{5}$.

Tucson Children Respiratory Study es un estudio longitudinal de patología respiratoria en
¿Cómo poder predecir si un lactante con

Síndrome Bronquial Obstructivo Recurrente será un futuro asmático?

Si un niño menor de 3 años presenta:

$+$

"Sibilancias frecuentes" ( $\geq 3$ episodios SBO/año) $\downarrow$

I criterio mayor ó 2 criterios menores

Tiene un "API positivo" y se puede asegurar con 77\% de certeza que ese niño va a padecer de asma a edad escolar (6-13 años). Si por el contrario el "API es negativo", con $68 \%$ de certeza se puede asegurar que los cuadros de bronquitis obstructiva o sibilancias van a desaparecer con el tiempo y ese niño no será un futuro asmático. Los niños con API positivo tienen 7 veces más riesgo de tener asma a edad escolar que los niños con API negativo.

\section{Criterios mayores:}

- Diagnóstico médico de eczema en los primeros 3 años de vida.

Antecedente de asma en alguno de los padres.

\section{Criterios menores:}

Diagnóstico médico de rinitis alérgica en los primeros 3 años de vida.

Sibilancias no asociadas a resfríos en los primeros 3 años de vida.

Eosinofilía periférica $\geq 4 \%$ en los primeros 3 años de vida.

Figura 1. Algoritmo Predictor de Asma. Fuente: CastroRodríguez J. Factores de riesgo para asma infantil. Neumología Pediatrica 2006; 1: 55-8.

niños, que incluyó 1.246 recién nacidos y sus familias (con 78\% elegibles) enrolados entre 1980 y $1984^{4}$. En esta cohorte se acuñó el índice IPA. Las variables utilizadas se eligieron como predictores significativos por análisis univariado. Sus componentes son: presencia de sibilancias frecuentes, asma activa, rinitis alérgica, eccema y eosinofilia ${ }^{4}$.

El índice IPA estricto incluye sibilantes frecuentes durante sus primeros tres años de vida como criterio mayor, y el laxo incluye haber tenido sibilancias antes de los tres años. El riesgo de desarrollar asma entre seis y once años es 4,3 a 9,8 veces más en un niño con IPA positivo si se utiliza el índice IPA estricto ${ }^{4}$. Los pacientes con IPA positivo tienen siete veces más riesgo de ser asmáticos en la edad escolar que aquellos con IPA negativo; Odds ratio (OR) 7,1 (intervalo de confianza o $\left.\mathrm{IC}_{95 \%} 3,5-14,1\right)^{1}$. Un índice IPA estricto se asocia a $77 \%$ de posibilidad de asma activa sobre los seis años ${ }^{5}$.

El índice IPA también se ha estudiado modificado, como el aplicado en 1954 niños de Leicester Respiratory Cohort, con seguimiento desde uno a diez años de edad. Para el IPA estricto se 
encontró un OR de asma a los siete años de 8,2 y un valor predictivo positivo (VPP) de $40 \%$. La eosinofilia en este estudio se cambió por sibilancias o tos desencadenada por alimentos. Los resultados mostraron conclusiones similares ${ }^{2}$. En todo caso, en estudios longitudinales se utiliza el índice IPA, no los índices modificados de IPA, para predecir asma ${ }^{4}$.

Existe un estudio aún en prensa, cuya cohorte concluye mejor likehood ratio (LR) de IPA modificado que IPA. Se enrolaron 289 recién nacidos desde noviembre de 1998 a mayo de 2000 de la cohorte COAST (Childhood Origins of ASThma), pero como requisito, al menos uno de los padres debía tener alergia respiratoria (uno o más aeroalergenos positivos en el prick test) y/o historia de diagnóstico médico de asma. Se incorporó sensibilización a uno o más aeroalergenos como criterio mayor, y sensibilización a leche, huevo o maní como criterio menor. El LR (+) de IPA fue 7,4 a los seis años, versus 21 en el IPA modificado ${ }^{8}$.

También se han acuñado otros índices predictivos de asma, por ejemplo el score de Isla de Wight del año 2003 en Inglaterra (con 1.456 niños) o el score PIAMA (Prevention and Incidence of Asthma and Mite Allergies), elaborado en 2009 en Holanda (con 3.963 niños) ${ }^{5}$. Entre los tres scores (PIAMA, IPA, y el de Wight), el IPA es el único evaluado en poblaciones diferentes y en estudios independientes, como estudios clínicos randomizados 5 .

Al evaluar periódicamente un paciente con riesgo de asma, se puede realizar mejor educación a la familia, y manejar más adecuadamente las descompensaciones y el estado basal del paciente. Los corticoides inhalatorios disminuyen hospitalizaciones, consultas al servicio de urgencia, síntomas, y mejoran pruebas de función pulmonar en pacientes pediátricos asmáticos ${ }^{9}$.

\section{Hipótesis}

Existe asociación entre Índice Predictivo de Asma (IPA) positivo en menores de 36 meses y presencia de asma entre los seis y siete años.

\section{Objetivos}

El objetivo general fue evaluar la asociación entre niños de seis y siete años de la comuna de Valdivia, con y sin diagnóstico de asma, y el resultado del índice predictivo de asma IPA positivo antes de los 36 meses. Los objetivos específicos fueron caracterizar las variables clínicas predictivas de asma definidas en el índice IPA, y describir si existen diferencias en resultados del índice IPA según sistema de atención, sea público o privado.

\section{Material y Método}

Se realizó un estudio de casos y controles, para estimar riesgo de una enfermedad de etiología multifactorial como es el asma.

\section{Definición de casos y controles}

Se consideraron casos a pacientes con diagnóstico clínico de asma actual, con domicilio en la comuna de Valdivia, con edad entre seis y siete años, atendidos en el Hospital Base Valdivia o en un centro privado de salud específico, independiente del resultado de índice IPA, hasta conseguir el número de casos requerido. Se agregó la consulta sobre antecedente de alergia alimentaria, como parte de anamnesis sobre atopía, y porque se ha incluido en otros estudios sobre predicción de asma. Se excluyeron pacientes con datos incompletos de encuesta IPA, con domicilio fuera de la comuna de Valdivia, pretérmino (menos de 37 semanas edad gestacional), o con diagnóstico médico de otra patología pulmonar crónica (displasia broncopulmonar, fibrosis quística, malformaciones pulmonares congénitas, daño pulmonar crónico, inmunodeficiencias). Controles se consideraron pacientes de mismo grupo etario $\mathrm{y}$ condiciones, pero sin asma actual.

Para descartar asma en pacientes que no la refieran o que no lo tenían claro, se aplicó el cuestionario ISAAC de pesquisa de asma. Las edades evaluadas corresponden a edad de seis a siete años, ya que son edades para las cuales el cuestionario ISAAC de asma está validado y fue aplicado en Chile (Figuras 2, 3 y 4).

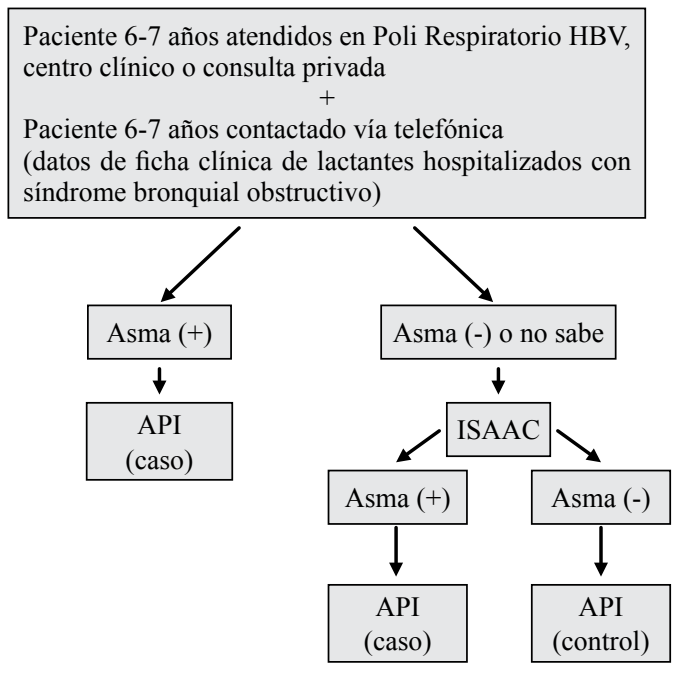

Figura 2. Esquema de definición de casos. HBV= Hospital Base de Valdivia. 
Fecha:

Procedencia:

- Edad en años cumplidos:

- Sexo: F ........ M .......

- ¿Tiene su niño/a diagnóstico de asma o está en estudio de esa enfermedad? Sí................

- ¿Tiene su niño/a alguna de las siguientes condiciones: prematurez, displasia broncopulmonar, fibrosis quística, malformaciones pulmonares congénitas, daño pulmonar crónico? Sí...............

\section{Encuesta API}

- ¿Alguno de los padres es asmático?

Ambos...... Ninguno....... Madre....... Padre......

Antes de los 3 años, su niño/a:

- Tuvo su hijo/a sibilancias frecuentes $(=0>3$ al año) (bronquitis obstructiva) Sí...... No.......

- ¿Tuvo sibilancias no asociadas a resfrío? (escucharle silbido al pecho o "gatitos") Sí....... No....... No se.......

- ¿Tuvo diagnóstico médico de eccema? Sí...... No....... No se.......

- ¿Tuvo diagnóstico médico de rinitis alérgica? Sí...... No...... No se.......

- ¿Tuvo hemograma con eosinófilos $>0=4 \%$ ? Sí....... No....... No se.......

- ¿Tuvo diagnóstico de alergia alimentaria (leche, huevo, maní...)? Sí...... No...... No se.......

Figura 3. Encuesta aplicada a casos y controles.

\section{CUESTIONARIO ISAAC ASMA PARA NIÑOS DE 6 A 7 AÑOS}

1. ¿Tuvo su hijo(a) alguna vez en su vida sibilancias o silbido al pecho (en cualquier época del pasado)?

$$
\text { Sí..... No.... }
$$

\section{SI CONTESTÓ NO SALTESE A LA PREGUNTA NÚMERO 6}

2. ¿Ha tenido su hijo(a) sibilancias o silbido al pecho en estos últimos 12 meses? Sí.....

No.....

SI CONTESTÓ NO SALTESE A LA PREGUNTA NÚMERO 6

3. ¿Cuantos ataques de crisis de sibilancias o silbido al pecho ha tenido en estos últimos 12 meses? Ninguno..... $\quad 1-3 \ldots . . \quad 4-12 \ldots . . \quad$ Más de $12 \ldots \ldots$

4. ¿En estos últimos 12 meses, cuantas veces se ha despertado en la noche debido a sibilancias o silbido al pecho?

Nunca.... Menos de una noche por semana.....

Una o más noches por semana.....

5. ¿En estos últimos 12 meses han sido las sibilancias o silbido al pecho tan severos (tan fuertes) como para no dejarlo hablar más de una o dos palabras entre cada respiración?

Sí..... No.....

6. ¿Ha tenido su hijo(a) asma alguna vez en su vida?
Sí.....
No.....

7. ¿En estos últimos 12 meses ha tenido sibilancias o silbido al pecho durante o después de hacer ejercicio (correr, etc)?

Sí..... No....

8. ¿En estos últimos 12 meses ha tenido tos seca en la noche? (aparte de la tos asociada a resfríos o infecciones respiratorias)

Sí.... No....
Figura 4. Cuestionario ISAAC para asma. Fuente: http://isaac.auckland.ac.nz/resources/ tools.php?menu=tools1. 


\section{Selección de casos y controles}

Se consideraron pacientes de edad entre seis y siete años, que fueron pesquisados al consultar en el Policlínico Respiratorio Infantil del Hospital Base Valdivia, o que fueron hospitalizados en el Hospital Base Valdivia, Subdepartamento de Pediatría, o atendidos en un centro de atención primaria determinado, o evaluados en la consulta privada incluida. Se les consideró caso si tenían asma y se aplicó el índice IPA. Si no tenían asma, se consideraron controles y también se les aplicó el índice IPA.

También se revisaron fichas clínicas de pacientes que estuvieron hospitalizados en la Unidad de Lactantes del Hospital Base Valdivia por síndrome bronquial obstructivo y que actualmente tenían entre seis y siete años. Se completaron datos con encuesta ISAAC para evaluar asma actual, vía telefónica. Luego se aplicó el índice IPA (si tenían asma se catalogaron como casos, si no la tenían se catalogaron como controles). Los encuestadores fueron un pediatra general, y dos pediatras broncopulmonares.

\section{Tamaño de la muestra}

Basado en el Censo de Población 2002 en que se estimó una población en la comuna de Valdivia de 140.559 habitantes de los que 34.050 eran menores de 15 años ${ }^{10}$, y de acuerdo con el reporte del estudio ISAAC en Chile en que se estimó una prevalencia de asma promedio de $18 \%$ en Valdivia en pediatría, que varía según grupo etario. La prevalencia de sibilancias en los últimos 12 meses fue $21,4 \%$ en el grupo entre seis y siete años, y $16 \%$ entre trece y catorce años ${ }^{5}$.

Se estimaron valores para casos y controles, una frecuencia de exposición entre los casos de 0,36 y frecuencia de exposición entre controles de 0,18. Empleamos valores convencionales de potencia o poder de $80 \%$ y para error tipo I $(\alpha)$ de $0,05 \%$, el tamaño de la muestra requerido fue de 94 casos, lo que se aproximó a 100. Se determinó una relación de caso:control de 1:1. Se usó el programa nQuery Advisor.

\section{Obtención y análisis de la información}

Se obtuvieron datos de la encuesta IPA y cuestionario ISAAC, en conversación directa con los padres o vía telefónica. Se solicitó consentimiento informado simple como parte de la aplicación de encuestas (IPA, ISAAC). Se obtuvo la información durante 14 meses, desde diciembre 2011. El estudio fue aprobado por el Comité de Ética Facultad Medicina de la Universidad Austral de Chile.

\section{Definición de variables}

Sibilancias frecuentes se consideró mayor o igual a cuatro crisis en un año. Dermatitis atópica/eccema si tenía diagnóstico médico. Alergia alimentaria si había el antecedente anamnéstico de síntomas respiratorios o cutáneos por comer leche, huevo o maní. Rinitis alérgica si tenía diagnóstico médico. Sibilancias no asociadas a resfrío si tenía sibilancias incluso sin estar cursando un resfrío. Eosinofilia si presentaba eosinófilos mayores o iguales a $4 \%$ en el hemograma antes de los tres años de vida. Asma si tenía diagnóstico médico o encuesta ISAAC positiva para asma.

\section{Análisis}

Los datos se digitaron primariamente en MS Excel y luego se analizaron mediante el programa STATA vs 11 (2009). Para conocer si existía o no relación entre variables se utilizó el test exacto de Fisher. Se consideró el valor de " $p$ " significativo si era $<0,05$. También se calculó el OR de las variables por separado y del índice IPA, en forma global y según sistema de atención (sistema público o privado). Finalmente se evaluó el índice IPA en análisis uni y multivariado de sus componentes.

\section{Aspectos éticos}

El estudio identificó algunos pacientes asmáticos sin diagnóstico previo, o sin el manejo adecuado (en mal control), a los que se les indicó acudir a evaluación por pediatra.

\section{Resultados}

Se entrevistaron pacientes entre seis y siete años con domicilio en la comuna de Valdivia, 101 con asma actual, 100 sin asma. Se comenzó a entrevistar a los pacientes en forma aleatoria hasta completar el número requerido, descartando aquellos con información incompleta. De la muestra de pacientes, $54,5 \%$ de los casos y un $47 \%$ de los controles, eran hombres. El porcentaje de pacientes entre seis y siete años fue similar en ambos grupos (Tabla 1).

Las frecuencias de cada uno de los parámetros necesarios para el índice IPA, se presentan en la Tabla 2. Hubo diferencias significativas $(p<0,001)$ entre ambos grupos en todas las variables que componen los criterios del índice IPA.

El 46,5\% de los asmáticos tenía antecedentes de asma en sus padres, destacando que un $24,8 \%$ de los casos tenía sólo madre asmática, significativamente más que si la herencia era sólo paterna $(12,9 \%)$ (Test Exacto de Fisher 0,006).

Un $86,1 \%$ de los asmáticos tuvo sibilancias 
Tabla 1. Caracterización general de casos (asmáticos) y controles (no asmáticos)

\begin{tabular}{|lccccc|}
\hline & Casos $(\mathbf{n}=\mathbf{1 0 1})$ & $\mathbf{\%}$ & Controles $(\mathbf{n}=\mathbf{1 0 0})$ & $\mathbf{\%}$ & $\mathbf{p}$ \\
\hline Sexo & & & & & \\
$\quad$ Masculino & 55 & 54,5 & 47 & 47 & $0,325^{*}$ \\
$\quad$ Femenino & 46 & 45,5 & 53 & 53 & \\
Edad (años) & & & & & \\
$\quad 6$ & 54 & 53,5 & 57 & 57 & $0,671^{*}$ \\
7 & 47 & 46,5 & 43 & 43 & \\
Sistema de atención & & & & & \\
$\quad$ Público & 53 & 52,5 & 65 & 65 & $0,086^{*}$ \\
$\quad$ Privado & 48 & 47,5 & 35 & 35 & \\
\hline
\end{tabular}

*Test exacto de Fisher.

Tabla 2. Distribución de casos y controles según variables consideradas en índice predictivo de asma: IPA

\begin{tabular}{|c|c|c|c|c|c|}
\hline & $\operatorname{Casos}(n=101)$ & $\%$ & Controles $(n=100)$ & $\%$ & $\mathbf{p}$ \\
\hline \multicolumn{6}{|c|}{ Sibilancias frecuentes } \\
\hline Sí & 87 & 86,1 & 12 & 12 & \multirow{2}{*}{$<0,001^{*}$} \\
\hline No & 14 & 13,9 & 88 & 88 & \\
\hline \multicolumn{6}{|c|}{ Sibilancias sin resfrío } \\
\hline Sí & 84 & 83,2 & 34 & 34 & \multirow[t]{2}{*}{$<0,001^{*}$} \\
\hline No & 17 & 16,8 & 66 & 66 & \\
\hline \multicolumn{6}{|l|}{ Eccema } \\
\hline Sí & 50 & 49,5 & 10 & 10 & \multirow[t]{2}{*}{$<0,001^{*}$} \\
\hline No & 51 & 50,5 & 90 & 90 & \\
\hline \multicolumn{6}{|c|}{ Rinitis alérgica } \\
\hline Sí & 55 & 54,5 & 14 & 14 & \multirow{2}{*}{$<0,001^{*}$} \\
\hline No & 46 & 45,5 & 86 & 86 & \\
\hline \multicolumn{6}{|c|}{ Eosinofilia } \\
\hline Sí & 25 & 24,8 & 6 & 6 & \multirow[t]{2}{*}{$<0,001^{*}$} \\
\hline No & 76 & 75,2 & 94 & 94 & \\
\hline \multicolumn{6}{|c|}{ Alergia alimentaria } \\
\hline Sí & 10 & 9,9 & 1 & 1 & \multirow[t]{2}{*}{$0,001 *$} \\
\hline No & 91 & 90,1 & 99 & 99 & \\
\hline \multicolumn{6}{|c|}{ Asma padres } \\
\hline Ambos & 9 & 8,9 & 0 & 0 & \multirow[t]{4}{*}{$<0,001^{*}$} \\
\hline Madre & 25 & 24,8 & 1 & 1 & \\
\hline Padre & 13 & 12,9 & 8 & 8 & \\
\hline No & 54 & 53,5 & 91 & 91 & \\
\hline
\end{tabular}

*Test exacto de Fisher.

frecuentes antes de los tres años, comparado con un $12 \%$ de los no asmáticos.

$\mathrm{Al}$ integrar las variables en el índice IPA, hubo $72,3 \%$ de paciente asmáticos que tenían índice positivo, y $3 \%$ en los no asmáticos (Tabla 3 ). Al realizar análisis univariado, lo más significativo se refiere a haber tenido sibilancias frecuentes antes de los tres años. OR 45,57 ( IC $_{95 \%}$ 19,95104,09) $(\mathrm{p}<0,001)$.
Si evaluamos nuestros pacientes comparando sistemas de salud, se desprende que el IPA positivo provenía principalmente de pacientes del sistema privado. (62,3\% de los pacientes asmáticos del sistema público tenía IPA positivo; $83,3 \%$ de los provenientes de sistema privado). No se logró comparar valores de OR respecto a IPA positivo y negativo según origen público o privado de los pacientes, porque no hubo controles en el sistema 
Tabla 3. Distribución de casos y controles, según el índice predictivo de asma-IPA y sistema de atención

\begin{tabular}{|c|c|c|c|c|c|}
\hline & $\begin{array}{c}\text { Casos } \\
(n=101)\end{array}$ & $\begin{array}{c}\% \\
\text { casos }\end{array}$ & $\begin{array}{c}\text { Controles } \\
(\mathbf{n}=100)\end{array}$ & $\begin{array}{c}\% \\
\text { controles }\end{array}$ & $\begin{array}{c}\text { OR } \\
\text { (IC 95\%) }\end{array}$ \\
\hline \multicolumn{6}{|l|}{ Sistema público } \\
\hline IPA+ & 33 & 62,3 & 2 & 3 & \multirow{3}{*}{$\begin{array}{c}34,1 \\
(8,9-185,1)\end{array}$} \\
\hline IPA- & 20 & 37,7 & 63 & 97 & \\
\hline Total sistema público & 53 & 100 & 65 & 100 & \\
\hline \multicolumn{6}{|l|}{ Sistema privado } \\
\hline IPA + & 40 & 83,3 & 0 & 0 & \multirow[t]{3}{*}{-} \\
\hline IPA- & 8 & 16,7 & 35 & 100 & \\
\hline Total sistema privado & 48 & 100 & 35 & 100 & \\
\hline \multicolumn{6}{|c|}{ Global (sistema público y privado) } \\
\hline IPA+ & 73 & 72,3 & 3 & 3 & \multirow{3}{*}{$\begin{array}{c}84,3 \\
(24,1-436,5)\end{array}$} \\
\hline IPA- & 28 & 27,7 & 97 & 97 & \\
\hline Total & 101 & 100 & 100 & 100 & \\
\hline
\end{tabular}

privado con IPA positivo (por lo tanto, contamos con un valor cero en ese cuadrante). Aún así se puede inferir que el OR del sistema privado es mayor, porque el público es sólo 34,1 y el global 84,3 .

En nuestro grupo de pacientes, la probabilidad de que un niño tenga asma es al menos 24 veces mayor si tenían índice IPA positivo (OR 84,3, $\left.\mathrm{IC}_{95 \%} 24,67-288,05\right)$.

En el análisis ajustado por componentes del IPA el mayor riesgo asociado a asma fue el asociado a sibilancias frecuentes (OR $36 \mathrm{IC}_{95 \%} 12,1$ 107,3) (Tabla 4).

\section{Discusión}

La herencia materna es más determinante que la paterna. La elevación de IgE materna se asocia a elevación en el cordón y a los seis meses (no así la elevación paterna $)^{11}$. En nuestros pacientes, también se observó más correlación de asma con antecedentes maternos que paternos. El riesgo de padecer asma se modifica con la regulación epigenética, la metilación de genes críticos puede inducir polarización a favor o en contra del fenotipo alérgico ${ }^{12}$.

Pareciera que en niños genéticamente predispuestos, la exposición y sensibilización a ácaros constituye el factor principal para desarrollar asma alérgica perenne ${ }^{13}$. Nuestro ambiente local es propicio para los ácaros, y es más frecuente encontrarlos en ambientes alfombrados. Esto se ve reflejado en un estudio basado en The ISAAC
Tabla 4. Análisis multivariado de las variables del índice predictivo de asma IPA

\begin{tabular}{|lrc|}
\hline Variable & OR & $\begin{array}{c}\text { Intervalo de } \\
\text { confianza 95\% }\end{array}$ \\
\hline Asma en padres & 1,34 & $(0,67-2,67)$ \\
\hline Sibilancias frecuentes & 36,06 & $(12,11-107,33)$ \\
\hline Sibilancias sin resfrío & 6,30 & $(2,12-18,70)$ \\
\hline Eccema & 10,10 & $(2,85-35,78)$ \\
\hline Rinitis alérgica & 3,75 & $(1,20-11,66)$ \\
Eosinofilia & 1,40 & $(0,27-7,35)$ \\
\hline Alergia alimentaria & 12,90 & $(0,32-511,03)$ \\
\hline
\end{tabular}

Europe Biomed Project del año 2004, para evaluar concentración de ácaros en domicilio, en siete países. De los lugares evaluados, Valdivia fue el segundo con más concentración de ácaro del polvo ${ }^{14}$.

En el año 2011 se estudiaron las características de un grupo de pacientes asmáticos de Valdivia (188 casos, 294 controles). De las diferencias estadísticamente significativas entre pacientes asmáticos y no asmáticos destaca mayor exposición a hongos o humedad en el hogar (OR 1,87 IC $_{95 \%}$ $1,18-2,97)$, y se concluye que $80 \%$ de nuestros pacientes con asma estaban sensibilizados a uno o más alergenos ${ }^{15}$, similar a lo encontrado por Calvo y cols., el año 2005, cuando se evaluaron 100 pacientes asmáticos con prick test, encontrando $100 \%$ de los pacientes con asma persistente 
severa positivos a ácaros, 85 y $73 \%$ para asma moderada y leve, respectivamente $(\mathrm{p}<0,01)^{13}$.

Nuestro estudio muestra un riesgo de desarrollar asma entre seis y siete años en lactantes con IPA positivo de al menos 24 veces más que aquellos con IPA negativo. Nuestra muestra incluye pacientes ya asmáticos, con heterogeneidad en cuanto al sistema de salud, así abarcamos distintas realidades. Consideramos pacientes de consulta privada, de nivel socioeconómico medio-alto, y eventualmente, con mayor porcentaje de asma atópica. Esto pudiera explicar los datos obtenidos por el índice IPA en nuestro medio, además de las condiciones ambientales de Valdivia. Es posible también que nos encontremos en transición como país hacia el desarrollo, con más casos de asma atópica que en el pasado.

Se considera que el aumento del asma está relacionado con cambios en la dieta materna, aumento del crecimiento fetal, reducción en el tamaño familiar, disminución de las infecciones en la infancia, aumento del uso de antibióticos e inmunizaciones ${ }^{5}$.

Nos quisimos enfrentar a una población específica, pacientes ya asmáticos, que fueron evaluados en forma retrospectiva, por lo que en ellos se infiere que debieran tener mayor positividad del IPA. Lo anterior puede significar un sesgo que aumente la magnitud de asociación, aunque nos pareció interesante evaluar IPA desde este otro punto de vista, vale decir, que hubiera sucedido si a los pacientes con asma diagnosticada, se les hubiera aplicado el índice IPA antes de los tres años de edad. Nuestros resultados nos permiten plantear que los pacientes con asma son adecuadamente pesquisados por un IPA positivo en el menor de tres años.

Es necesario señalar las limitaciones del estudio, siendo la principal su diseño retrospectivo, el aplicar el índice IPA en base a recuerdos de los cuidadores, sobre síntomas hace 3-4 años, pero así reafirmamos la utilidad de este índice, porque aún evaluados retrospectivamente, la diferencia entre los positivos, asmáticos y no asmáticos, fue estadísticamente significativa. Además hubo escasa interacción con los padres, lo que podría haber dificultado las respuestas. Otro punto a considerar es que como los encuestadores incluyeron a especialistas, pudieron haber pesquisado más pacientes asmáticos, que en otras condiciones habrían resultado subdiagnosticados.

\section{Conclusión}

De acuerdo a esta experiencia, el índice IPA, como herramienta para predecir asma, es aplicable a nuestra población. Incluso en forma retrospectiva se asocia más a pacientes asmáticos que no asmáticos.

En cuanto a los componentes del score IPA, la variable que más se asocia a asma entre seis y siete años es tener sibilancias frecuentes antes de los tres años. Encontramos más IPA positivo en pacientes provenientes del sector privado, pudiendo esto deberse a múltiples causas a estudiar, incluyendo aspectos clínicos y ambientales.

\section{Bibliografía}

1.- CASTRO-RODRÍGUEZ J. Factores de riesgo para asma infantil. Neumología Pediatrica 2006; 1: 55-8.

2.- CASTRO-RODRÍGUEZ J, HOLBERG C, WRIGHT A, MARTÍNEZ F A. Clinical Index to Define Risk of Asthma in Young Children with Recurrent Wheezing. Am J Respir Crit Care Med 2000; 162: 1403-6.

3.- ASTUDILlO P. Asma infantil en Chile. Neumol Pediatr 2006; 1: 91-3.

4.- CASTRO-RODRÍGUEZ J. The Asthma Predictive Index: A very useful tool for predicting asthma in young children. J Allergy Clin Immunol 2010; 126: 212-6.

5.- MALLOL J, AGUIRRE V, AGUILAR P, CALVO M, AMARALES L, ARELLANO P, et al. Cambios en la prevalencia de asma en escolares chilenos entre 1994 y 2002. International Study of Asthma and Allergies in Childhood (ISAAC) Chile phases I and III. Rev Med Chile 2007; 135: 580-6.

6.- The International Study of Asthma and Allergies in Childhood Disponible en: http://isaac.auckland.ac.nz/ Consultado el 11 de enero de 2013.

7.- MALLOL J, CORTEZ E, AMARALES L, SÁNCHEZ I, CALVO M, SOTO S, et al. Prevalencia del asma en escolares chilenos. Estudio descriptivo de 24.470 niños. ISAAC-Chile. Rev Med Chile 2000; 128: 279-85.

8.- CHANG T, LEMANSKE R, GUILBERT T, GERN J, COEN M, EVANS M, et al. Evaluation of the Modified Asthma Predictive Index in High-Risk Preschool Children. J Allergy Clin Immunol Pract 2013; 1: 152-6.

9.- LINUS N. Corticoides Inhalados. Neumol Pediatr 2006; 1: 73-6.

10.- http://reportescomunales.bcn.cl/2012/index.php/Valdivia/ Consultado: el 11 de febrero de 2013.

11.- CASTRO-RODRÍGUEZ J. The Asthma Predictive Index: early diagnosis of asthma. Allergy Clin Immunol 2011; 11: 157-61.

12.- MILLER R, SHUK-MEI H. Environmental Epigenetics and Asthma. Current Concepts and Call for Studies. Am J Respir Crit Care Med 2008; 177: 567-73.

13.- CALVO M, FERNÁNDEZ-CALDAS E, ARELLANO P, MARÍN F, CARNÉS J, HORMAECHEA A. Mite allergen exposure, sensitisation and clinical symptoms 
in Valdivia, Chile. J Invest Allergol Clin Immunol 2005; 15: 189-96.

14. WICKENS K, DE BRUYNE J, CALVO M, CHOONKOOK S, JAYARAJ G, LAI C, et al. The determinants of dust mite allergen and its relationship to the prevalence of symptoms of asthma in the Asia-Pacific region.
Pediatr Allergy Immunol 2004; 15: 55-61.

15.- BONEBERGER A, HAIDER D, BAER J, KAUSEL L, VON KRIES R, KABESCH M, et al. Environmental Risk Factors in the First Year of Life and Childhood Asthma in the Central South of Chile. J Asthma 2011; 48: 464-9.

Correspondencia a:

Dra. Evelyn Krause Grob

Instituto de Pediatría, Universidad Austral de Chile,

Valdivia.

Email: evelyn_kr@hotmail.com 